\title{
Respiratory Research in the Critically Ill Pediatric Patient: Why Is It So Difficult?
}

\author{
Martha AQ Curley RN PhD
}

\author{
Introduction \\ Why Are Large-Scale Prospective Randomized Controlled Clinical Trials \\ Lacking in Pediatrics? \\ Hurdles Faced By Clinical Trialists \\ Study Design \\ Subject Enrollment \\ Protocol Adherence \\ Sustained Commitment \\ Suggestions for Improved Success of Future Clinical Studies \\ Including Children \\ Restructuring the Clinical Research Enterprise \\ Alternative Research Designs \\ Building Research Capacity \\ Summary
}

Pediatric clinicians strive to base their management decisions on best available evidence. In the quantitative research paradigm, the highest level of evidence is derived from a conclusive randomized controlled clinical trial (RCT). Currently, there are few adequately powered RCTs to support pediatric acute respiratory care, but this landscape is changing. We are all obligated to ensure the relevance of our research, to mentor junior investigators, and to support knowledge development in our field. This paper reviews the hurdles faced by clinical investigators in the field of pediatric critical care and offers suggestions for future clinical studies. Key words: multicenter trial; clinical research; collaborative research; mechanical ventilation. [Respir Care 2011;56(9):1247-1254. (C) 2011 Daedalus Enterprises]

\section{Introduction}

What is optimal exhaled tidal volume in a ventilated 2-month old with respiratory syncytial virus? Should we routinely instill saline while suctioning a patient with 3.5-mm endotracheal tube? Does $45^{\circ}$ head elevation prevent ventilator-associated pneumonia in a toddler? Should neuromuscular blockade be used to shorten the duration of mechanical ventilation in a 6-year-old asthmatic with mul-

\footnotetext{
The author has disclosed no conflicts of interests.

Correspondence: Martha AQ Curley RN PhD, School of Nursing, University of Pennsylvania, Claire M Fagin Hall, 418 Curie Boulevard, Philadelphia PA 19104. E-mail: curley@ nursing.upenn.edu.
}

DOI: $10.4187 /$ respcare. 01255 
tiple air leaks? How can we best maintain lung volume when suctioning a child supported on high-frequency oscillatory ventilation? These questions, often posed during multidisciplinary rounds, lack sufficient evidence to answer.

When caring for critically ill pediatric patients, clinicians strive to base their management decisions on best available evidence. In the quantitative research paradigm, the highest level of evidence is derived from a conclusive randomized controlled clinical trial (RCT) where the lower confidence interval exceeds the minimally clinically important benefit. We know that changes in management strategies should be data-driven and then systematically evaluated. That said, there are few adequately powered RCTs to support the rapid-fire clinical decisions that hallmark the practice of pediatric critical care. Pulmonary critical care research literature is definitely more robust for adult patients than for infants and children. ${ }^{1}$

Given the lack of pediatric-specific data, clinicians caring for critically ill infants and children have learned to practice without it. Pediatric clinicians are early yet selective adopters of interventions that have been primarily tested in the adult and/or neonatal patient population. ${ }^{2}$ Once adopted, clinicians quickly lose equipoise on whether the intervention offers the pediatric patient clinical benefit. Equipoise, or genuine uncertainty within the expert community on whether an intervention is beneficial, provides the ethical basis for patient randomization to different treatment arms of a clinical trial. ${ }^{3}$ Once an expert community loses equipoise, randomization becomes unethical. So why is respiratory research in pediatric acute care lacking? This paper will discuss why these data are limited and review the hurdles faced by clinical trialists in the field of pediatric respiratory acute care. The paper concludes by making recommendations for improved success for future clinical trials.

\section{Why Are Large-Scale Prospective RCTs Lacking in Pediatrics?}

Large-scale prospective RCTs are currently lacking in pediatrics because, although substantial progress has been made in the last decade, the pediatric critical care community does not have a mature coordinated system to support the recruitment of large numbers of subjects within a short period of time to reasonably answer a research question. Definitive trials typically require hundreds if not thousands of patients to achieve adequate power. Compared to the adults and neonates, there are simply fewer pediatric patients with acute lung injury (ALI). ${ }^{4}$ In addition, the etiology of pediatric ALI is diverse, the duration of mechanical ventilation is shorter, mortality is infrequent, and a high proportion of these children have a wide-range of pediatric-specific comorbid conditions. ${ }^{1,2,5,6}$
Zimmerman and colleagues conducted the first population-based, prospective cohort study designed to determine the population incidence and outcomes of pediatric ALI. ${ }^{4}$ They calculated an incidence of 12.8 cases per 100,000 person-years, and an in-hospital mortality rate of $18 \%$. There were no statistically significant associations between age, sex, risk factors, and outcomes. For comparison, the adult King County Lung Injury Project reported teen (15-19 years) and adult ALI incidences of 16.0 and 78.9 cases per 100,000 person-years and in-hospital mortality rates of $24.0 \%$ and $38.5 \%$, respectively. ${ }^{7}$

The most common respiratory conditions in pediatrics include pneumonia and pulmonary edema/effusion. ${ }^{1}$ Mortality is a rare event in this prevalent patient group, unless the patient is immunocompromised. ${ }^{4}$ Oncologic diagnoses are uncommon (approximately 3\%) but are disproportionally represented in pediatric acute respiratory distress syndrome (ARDS) (approximately 13\%) and in the mortality cohort $(>40 \%) .5,8$ Power calculations must accommodate these nuances and center on clinically important outcomes: specifically, outcomes important enough to convince clinicians to change their practice.

The accrual of sufficient numbers of diverse participants in the shortest period of time mandates multicenter research. The duration of the clinical trial is especially important in the acute care environment, where the threat of secular change is high. Unlike bench research, it is impossible to control all the clinical interventions applied to patient care, and, at the very least, the care provided to subjects at the start of a clinical trial should match the care provided to subjects at the end of the clinical trial.

Santschi and colleagues recently published the results of the Pediatric Acute Lung Injury Ventilation (PALIVE) study. ${ }^{1}$ The purpose of PALIVE was to describe mechanical ventilation strategies used in children with ALI/ARDS in a large representative sample of North American and European pediatric intensive care units (PICUs) and to determine the feasibility of performing a large intervention study in children with ALI/ARDS. With 3,823 patients screened, $54 \%$ of patients were ventilated, and $11 \%$ were diagnosed with ALI, but only $4.3 \%$ of patients met standard inclusion/exclusion criteria and therefore would have been eligible for enrollment in a pediatric ALI/ARDS clinical trial. They estimated that it would take 4 years and about 60 PICUs to enroll 800 children with ALI/ARDS, aiming for a reduction in mortality as the primary end point. Using a nonmortality primary outcome measure, such as ventilator-free days or post-discharge functional status, may improve the power.

Multicenter clinical trials are complex. Investigatorinitiated RCTs are typically conducted as the capstone project within a program of research. Marshall and Cook describe a program of research as involving multiple steps that ends with the translation of study findings into clinical 
practice. ${ }^{9}$ They note that clinical research is undertaken to resolve uncertainty. Research programs define the uncertainty, establish relevance, and determine the feasibility of answering the research question.

Research programs start with a systematic review or meta-analysis of what is known about the research question. ${ }^{9}$ They gather information on the prevailing attitudes about the phenomena of concern, by surveying clinicians through questionnaires. They include observational studies to describe the practice variability that actually occurs in day-to-day practice. They pilot-test the proposed study protocol to evaluate its feasibility, acceptability, and adherence rates. They then design an RCT based upon what was learned in the previous steps. RCTs require epidemiologic and descriptive studies to guide trial design. ${ }^{10}$

Multicenter RCTs require a well coordinated system and funding mechanism. Pediatrics has led the critical care field in leveraging teams though the Pediatric Acute Lung Injury and Sepsis Investigators (PALISI) network. The PALISI Network was founded in 2002, when pediatric critical care investigators participating in 3 multicenter trials combined to form one combined research network. ${ }^{11-13}$ Now, over 80 PICUs have joined together to identify optimal supportive, preventive, and therapeutic strategies for acute life-threatening pulmonary syndromes that affect infants and children. The network's goal is to perform multicenter research studies to better describe disease processes and outcomes in pediatric patients and to evaluate interventions in this population.

From a funding perspective, pediatric critical care medicine found a home at the National Institutes of Health (NIH) when, in 2005, the National Institute of Child Health and Human Development formed the Collaborative Pediatric Critical Care Research Network (http://www.nichd. nih.gov/research/supported/cpccrn.cfm). The network consists of 6 clinical centers, chosen on the basis of proposed scientific work, patient ethnicity, and concordance with programmatic objectives. The goal of the network is to develop an infrastructure to pursue well designed collaborative clinical trials and meaningful descriptive studies in pediatric critical care medicine (http://www.cpccrn.org). The National Institute of Child Health and Human Development has had a history of funding pilot studies in pediatric respiratory failure.

Building a program of research takes time, persistence, and funding. The pediatric prone positioning study provides a point of reference. Curley and others published a systematic review of prone positioning patients with ARDS in $1999,{ }^{14}$ a single-center pilot study of early and repeated prone positioning in pediatric patients with ALI in 2000, ${ }^{15}$ then the results of a multicenter RCT of prone positioning in children with ALI in 2005. ${ }^{12}$ The American Association of Critical Care Nurses and the Society of Critical Care Medicine funded the pilot study and the National Institutes of Nursing Research funded the RCT (RO1NR05336). The research team included respiratory therapists, nurses, and pediatric intensivists. The PALISI network was used to recruit participating sites.

\section{Hurdles Faced By Clinical Trialists}

Obstacles faced by pediatric clinical trialists include designing a robust research study, enrolling a sufficient number of subjects into the clinical trial to answer the research question, assuring local adherence to the research proto$\mathrm{col}$, and sustaining the commitment and collaboration of multiple investigators and clinical sites over the study period. ${ }^{16}$ In designing clinical trials there are few absolute right or wrong activities, but a series of important tradeoffs. Clinical trialists balance what is feasible in conducting a trial with what will be generalizable to PICU practice.

\section{Study Design}

The research design must be robust to accommodate the clinical environment and answer the research question. Core design elements, embedded in the research question, include delineating the patient situation/problem and a clinically important outcome.

Inclusion and exclusion criteria are crafted to describe the desired patient situation of concern. The "pediatric" age group expected to be enrolled in a clinical trial may appear obvious, but trialists have struggled with the operational definition of the "pediatric" patient. Pediatric ICUs typically admit patients across the age and developmental spectrum. Pediatric trialists enroll infants, not expected to exhibit neonatal physiology, through adolescents, not expected to exhibit mature physiology. Anticipating a developmental impact on a study's outcome, all age groups are typically included in pediatric RCTs, then age cohorts are controlled statistically during the data analysis phase. This improves the generalizability of study results to the entire PICU population.

The etiology of ALI/ARDS is diverse, and although substantial progress has been made in operationalizing ALI and ARDS, targeting interventions to specific disease entities may be beneficial. Patients with markedly different pathophysiology, for example, patients with airway obstruction, cyanotic heart disease, progressive neuromuscular weakness, and pulmonary hypoplasia should be studied as separate diagnostic subgroups. ${ }^{5}$ Patients with direct/ indirect ALI can be enrolled into a pediatric RCTs, then disease cohorts can be evaluated in post-hoc analyses.

Using mortality as a primary outcome variable is not practical in pediatric ALI/ARDS trials. ${ }^{4}$ An impossible number of subjects would be required to demonstrate effect. Attributing death to ALI/ARDS, as opposed to un- 
derlying disease, is also difficult, as most children with ARDS have comorbid conditions that are unique and different than those of adults. Mortality is also insensitive to other important clinical outcomes, for example, survival with substantial neurologic morbidity.

Alternative end points such as ventilator-free days ${ }^{17}$ are used, and under appropriate circumstances, major morbidities could also be considered as primary end points. ${ }^{18}$ Measures of morbidity include composite end points such as time-to-resolution of organ failure. To support this work, validated organ dysfunction scores have been developed for children. ${ }^{19,20}$ In addition, we know that adult survivors of critical illness suffer a decline in health-related quality of life ${ }^{21}$ but comparable pediatric data do not exist. Describing long-term outcomes, including functional ${ }^{22}$ or neurocognitive health, quality of life, and economic burden after pediatric critical illness, would also benefit the field.

\section{Subject Enrollment}

There is substantial variation in patient populations across clinical sites. ${ }^{5}$ Knowledge of local referral patterns is essential when recruiting site participation in clinical trials. Data points can include a PICU's recent history of subject enrollment in a clinical trial enrolling a similar patient population, or asking the site to complete mock screening for at least one month. Although seasonal variation will impact screening in any month, asking a site to prospectively apply inclusion/exclusion criteria allows a fair representation of the projected enrollment from candidate sites. These data, collected in a de-identified manner, are considered preparatory for research and, depending on the local institutional review board (IRB), may be considered an activity that does not require local IRB approval.

All sites participating in a multi-site clinical trial must use the same protocol. Permitting substantial variation in what happens to subjects at different sites introduces bias and risks, rendering study results uninterpretable. The system for protecting research subjects was designed when most research studies took place at a single institution. With multi-site clinical research, the same protocol is reviewed multiple times. Menikoff ${ }^{23}$ noted that these duplicative reviews provide relatively few benefits and may reduce the likelihood that studies are in keeping with relevant ethical standards. To limit protocol drift between participating sites, coordinating centers should provide each participating sites with a template of a completed IRB application and informed consent form. Site co-investigators can then model their application and informed consent form on the template. All subsequent changes are reviewed and approved by the coordinating center.
Approximately half of all eligible patients are enrolled into clinical trials. The primary reason for this eligibilityenrollment gap is parent or legal guardian refusal. ${ }^{24}$ Parents are ethically entitled and legally empowered to make decisions on behalf of their children. ${ }^{25}$ There is site-to-site variability in consent rates, based on PICU and investigatorspecific characteristics. Obtaining informed consent is difficult, especially when enrollment is time-sensitive. The parent or legal guardian may not be physically or emotionally available. Jansen and others have argued that deferred consent may be justified in emergency critical care research. ${ }^{26}$

Federal regulations also require that the child, when cognitively capable, provide his or her assent to participate in research. While the age of assent varies on a local level, most IRBs require a plan on how assent will be obtained when enrolling children older than 7 years of age. When obtaining assent, the research and potential risks and benefits are explained using language that a child can understand. The readability statistics available on most word processing programs can help investigators level language to match the subject's age and cognitive capacity.

\section{Protocol Adherence}

Ensuring protocol adherence is critically important in multi-site clinical trials. Inter-site variation threatens a study's internal validity. All potential co-investigators, and their colleagues, must agree to follow the study protocol. Prior to inserting a protocol into a clinical environment, assumptions about the research environment require validation. Practice standards may vary across clinical settings, especially international settings. Practice surveys that include data elements that may impact a study's primary outcome should be completed at the start of, and periodically throughout the clinical trial. For example, if the primary outcome variable for a study is ventilator-free days, clinical trialists will want to know about each participating site's baseline ventilator-associated pneumonia (VAP) prevention practices and adherence rate, because VAP rate will impact ventilator-free days.

Subject safety requires that all clinicians demonstrate competence in the clinical protocol before enrolling subjects. Since different disciplines interact with a clinical protocol in varying ways, discipline-specific protocol "certification" and competence should be clearly established. Competence can be maintained by enrolling a minimum number of subjects each quarter, or by retraining.

Elements of a protocol that are critical to the validity of the study's conclusions are monitored and reported by site on a quarterly basis. Reports can be automatically extracted from existing case report forms, or specific queries can be sent to participating sites on a random basis. All 


\section{Respiratory Research in the Critically Ill Pediatric Patient: Why Is It So Difficult?}

Table 1. Competing Clinical Trials: Evaluating the Potential for Co-enrollment

Are the primary outcome variables different?

Is there a potential interaction effect on the primary outcome?

Can you statistically evaluate effect modification?

Can subject assignment be balanced?

Can attribution for adverse events be assigned?

How complex are the interventions? Can a clinical site safely do both clinical trials?

Will multiple consent processes overburden the family?

primary outcome data require source verification, which is completed during site visits.

\section{Sustained Commitment}

Clinical research teams work to sustain the commitment and collaboration of multiple disciplines over the study period. Sustaining enthusiasm over a long clinical trial is challenging. Conference calls help to bring investigators together to review study progress and collectively solve problems and address competing demands.

It is important to choose one's collaborators wisely. Collaborators should be experienced in clinical research and should be local leaders who can champion the project. Local investigators should have the ability to devote time to the clinical trial and have a history of successful collaboration. Potential collaborators should provide evidence of their capacity to enroll and safely manage a critically ill patient on a protocol. Optimally, each site should have a skilled study coordinator who is knowledgeable in the field, detail-oriented, tenacious, and single-minded.

Undoubtedly, clinical trials will overlap. ${ }^{27}$ Table 1 reviews factors that are considered when evaluating the capacity of several studies to enroll subjects from a single site. Intervention trials in the same patient population are avoided. When appropriate, sharing schemes are first agreed upon by each study's principal investigator. Recommendations for sharing schemes are then proposed to the clinical sites to ensure consistent decision making across all clinical sites. This process requires monitoring to ensure that the agreements are successfully implemented.

\section{Suggestions for Improved Success of Future Clinical Studies}

Strategies to improve our success in conducting clinical trials in pediatric respiratory acute care include changing our thinking about clinical research. For example, including children in larger clinical trials conducted in the adult or neonatal populations, restructuring the clinical research enterprise, using alternative research designs to answer clinical research questions, and building our research capacity.

\section{Including Children}

Fundamentally, we should challenge the convention of automatically excluding children from clinical studies conducted in adult or neonatal patients. Pediatric patients should be excluded when there are developmental differences and included when there are not. ${ }^{5}$ In 1998, the NIH mandated that investigators include children in adult trials unless their exclusion was clearly justified. ${ }^{28}$ Although several RCTs in adults have extended eligibility criteria to children 12 years of age, this maneuver has not provided the field with any new data to improve the care provided to pediatric patients with acute respiratory illnesses. ${ }^{29,30}$

Halpern and colleagues have challenged the notion that novel interventions be always tested in adults prior to children. ${ }^{31}$ They note that, although the practice may protect children from research risks, it also may threaten a child's well-being by depriving them of evidence to guide their care. Children are traditionally viewed as "participants of last resort," due to their vulnerability and decisional incapacity. Halpren et al note that RCTs should be conducted in children first when children are more apt to benefit and adults are more apt to be harmed.

Interest continues in identifying reliable biomarkers to both detect ALI and pace ALI resolution in response to a therapy. ${ }^{32,33}$ Ideal biomarkers that are sensitive and specific, reflecting disease progression and/or resolution, have yet to be validated. Similar to targeted therapy in oncology, Angus and others note that we are close to using theragnostics in critical care: specifically, using biomarkers to identify patients most likely to respond to an intervention. ${ }^{34}$ Once validated, we may be able to use biomarkers to adequately power smaller more feasible pediatric clinical trials.

\section{Restructuring the Clinical Research Enterprise}

The NIH recently highlighted the need to reinvigorate the biomedical research community through one of 5 key themes of the Director's Opportunity for Research award (RFA-OD-10-005). The award encouraged investigators to cultivate new collaborations by assembling multidisciplinary or interdisciplinary teams in conducting innovative research. The goal of the award was to strengthen research capacity and to enhance cross-fertilization of disciplines by recruiting new investigators and new expertise into the research community, and by developing and retaining these talents in a collaborative environment that fosters creativity and exploration.

We should think differently about how we organize ourselves around conducting RCTs. Clinical research should 
mirror clinical practice. We practice and should conduct clinical research within multidisciplinary teams: groups that share a collective mission designed to achieve shared goals. Leveraging "team" strengths (ie, respiratory therapist - nursing - medicine - pharmacist - physical therapist - nutritionist - economist team strengths) can unify us around clinical problems so that we can ask multiple primary and secondary questions within a single clinical trial. Most of us have witnessed stellar clinical outcomes when team members bring their best available disciplinary knowledge or disciplinary lens to the bedside. Similar results can occur when we join together to answer clinical research questions. This is not the traditional multidisciplinary framework where several disciplines work in parallel around a common problem, or the interdisciplinary framework where disciplines work jointly, sharing frameworks and methods. This new transdisciplinary framework may facilitate the creation of new knowledge using a new language and carries the potential for study findings to be enriched and be more rapidly translated into practice. Transdisciplinary frameworks will require full investigator partnership and meaningful methods to share effort, funding, and authorship.

For example, multidisciplinary innovation will continue to optimize the care provided to young patients with asthma. These patients require transitional care programs that span the continuum of care; they require novel interventions that bridge the work of bench and clinical scientists; they require economic modeling and re-evaluation; and they require both short-term and long-term outcome monitoring. Patients and families experience care that is tied to the collective output of all disciplines. Although we have made progress in conducting science in disciplinary silos, breakthrough science will require a transdisciplinary perspective.

\section{Alternative Research Designs}

Changing our approach to knowledge generation may require a paradigm shift in how we grade or stratify the quality of our research methods. Research methods are derived from the question posed. Understanding a patient's or family's perspective on a topic requires qualitative methodologies. Stellar examples of qualitative studies include Meert's program of research on end-of-life decision making. ${ }^{35-37}$ Qualitative research often forms the basis for subsequent quantitative study, for example, when describing and then evaluating patient reports of dyspnea.

In addition, a well structured factorial design allows investigators to test 2 interventions with only a marginal increase in sample size. ${ }^{27,30}$ The design offers a substantial benefit where multiple interventions need to be evaluated alone or in combination in the same population. ${ }^{25}$ Such designs have been successfully used by the Acute Respi- ratory Distress Syndrome Network and offer an efficient solution when exploring interventions that may be interactive.

Bayesian statistical methods, which leverage the power of an adult trial to an associated pediatric trial, are being considered with increased frequency. ${ }^{29,30}$ This approach links an a priori pediatric estimate of a treatment effect to previously or concurrently observed value in adults. Leveraging data from the adult trial substantially increases the power of the associated pediatric clinical trial. This statistical approach has the potential of making pediatric clinical trials feasible, because fewer pediatric subjects would be required to show efficacy.

\section{Building Research Capacity}

In the future we can expect more "virtual" research teams to come together to tackle clinical problems. Coprincipal-investigators can sit on opposite sides of the country and the world. Free cyberspace links provide limitless opportunities to connect beyond the expensive and rudimentary conference call.

Highly functional research teams should not disband at the end of an RCT, but should launch directly into another project. Using existing structures will abbreviate start-up time and costs. Joining preexisting networks will strengthen our capacity to respond to evolving events such as the H1N1 flu epidemic. For example, coordinating the efforts of the PALISI network with other successful research groups, including the Canadian Critical Care Trials Group, the Australian and New Zealand Intensive Care Society Clinical Trials Group, and the European Society of Pediatric and Neonatal Intensive Care (to name a few), would leverage our capacity to respond rapidly to international issues.

We can build research capacity within the field by capitalizing on transdisciplinary and interdisciplinary mentoring. Clinical scientists need not practice independently. Finding mentors and colleagues and building a team with expertise in experimental design, protocol implementation, statistical analysis, scientific writing, research ethics, and grant preparation would booster our research capacity.

When choosing a science mentor, one should look for synergy. ${ }^{38-40}$ You should have a real interest in your mentor's research, you should find their publications exciting, your mutual chemistry should feel right. Junior investigators need access to their mentors. Face time on a regular meeting schedule in person or electronically is important. Your mentor should help you launch your independent research career and provide you an opportunity to carve out your unique area of research. Stellar mentors are passionate about knowledge development. They are kind and truly interested in your work. They are generous and share their knowledge and experience. They give you their good 
ideas. They are inspiring, encouraging, humble, and your best advocate. Eventually you should be recognized as more expert than your mentor in your program of research.

Finally, finding the necessary funds to support clinical research continues to be challenging. Although "research" is often in the mission statement of many healthcare organizations, few are adequately structured to support clinical research. Indirect funds are often channeled to support the research enterprise that is dominated by laboratorybased research. Budgeting a clinical trial occurs concurrently with study design. Many professional organizations and philanthropic organizations have grant monies available for clinical projects, and some can be leveraged to support larger clinical trials.

\section{Summary}

In conclusion, pediatric clinicians strive to base their management decisions on best available evidence. In the quantitative research paradigm, the highest level of evidence is derived from a conclusive RCT. Currently, there are few adequately powered RCTs to support pediatric acute respiratory care, but this landscape is changing. We are all obligated to ensure the relevance of our research, to mentor junior investigators, and to support knowledge development in our field. Although this paper presents the major design and implementation challenges faced by clinical trialists in the field of pediatric acute respiratory care, nothing beats asking and answering clinical research questions that are important to vulnerable patient populations.

\section{REFERENCES}

1. Santschi M, Jouvet P, Leclerc F, Gauvin F, Newth CJ, Carroll CL, et al.; PALIVE Investigators; Pediatric Acute Lung Injury and Sepsis Investigators Network (PALISI); European Society of Pediatric and Neonatal Intensive Care (ESPNIC). Acute lung injury in children: therapeutic practice and feasibility of international clinical trials. Pediatr Crit Care Med 2010;11(6):681-689.

2. Khemani RG, Newth CJ. The design of future pediatric mechanical ventilation trials for acute lung injury. Am J Respir Crit Care Med 2010;182(12):1465-1474.

3. Freedman B. Equipoise and the ethics of clinical research. $\mathrm{N}$ Engl J Med 1987;317(3):141-145.

4. Zimmerman JJ, Akhtar SR, Caldwell E, Rubenfeld GD. Incidence and outcomes of pediatric acute lung injury. Pediatrics 2009;124(1): 87-95.

5. Randolph AG, Meert KL, O'Neil ME, Hanson JH, Luckett PM, Arnold JH, et al. The feasibility of conducting clinical trials in infants and children with acute respiratory failure. Am J Respir Crit Care Med 2003;167(10):1334-1340.

6. Khemani RG, Markovitz BP, Curley MA. Characteristics of children intubated and mechanically ventilated in 16 PICUs. Chest 2009; 136(3):765-771.

7. Rubenfeld GD, Caldwell E, Peabody E, Weaver J, Martin DP, Neff $\mathrm{M}$, et al. Incidence and outcomes of acute lung injury. N Engl J Med 2005;353(16):1685-1693.
8. Willson DF, Thomas NJ, Markovitz BP, Bauman LA, DiCarlo JV, Pon S, et al. Effect of exogenous surfactant (calfactant) in pediatric acute lung injury: a randomized controlled trial. JAMA 2005;293(4): 470-476.

9. Marshall JC, Cook DJ. Investigator-led clinical research consortia: the Canadian Critical Care Trials Group. Crit Care Med 2009;37(1 Suppl):S165-S172.

10. Nicholson CE, Gans BM, Chang AC, Pollack MM, Blackman J, Giroir BP, et al. Pediatric critical care medicine: planning for our research future. Pediatr Crit Care Med 2003;4(2):196-202.

11. Randolph AG, Wypij D, Venkataraman ST, Hanson JH, Gedeit RG, Meert KL, et al. Effect of mechanical ventilator weaning protocols on respiratory outcomes in infants and children: a randomized controlled trial. JAMA 2002;288(20):2561-1568.

12. Curley MA, Hibberd PL, Fineman LD, Wypij D, Shih MC, Thompson JE, et al. Effect of prone positioning on clinical outcomes in children with acute lung injury: a randomized controlled trial. JAMA 2005;294(2):229-237.

13. Willson DF, Thomas NJ, Markovitz BP, Bauman LA, DiCarlo JV, Pon $\mathrm{S}$, et al. Effect of exogenous surfactant (calfactant) in pediatric acute lung injury: a randomized controlled trial. JAMA 2005;293(4): 470-476.

14. Curley MAQ. Prone positioning of patients with acute respiratory distress syndrome: a systematic review. Am J Crit Care 1999;8(6): 397-405.

15. Curley MA, Thompson JE, Arnold JH. The effects of early and repeated prone positioning in pediatric patients with acute lung injury. Chest 2000;118(1):156-163.

16. Irving SY, Curley MAQ. Challenges to conducting multicenter clinical research: Ten points to consider. AACN Adv Crit Care 2008; 19(4):164-169.

17. Schoenfeld DA, Bernard GR; ARDS Network. Statistical evaluation of ventilator-free days as an efficacy measure in clinical trials of treatments for acute respiratory distress syndrome. Crit Care Med 2002;30(8):1772-1777.

18. Curley MA, Zimmerman JJ. Alternative outcome measures for pediatric clinical sepsis trials. Pediatr Crit Care Med 2005;6(3 Suppl): S150-S156.

19. Graciano AL, Balko JA, Rahn DS, Ahmad N, Giroir BP. The Pediatric Multiple Organ Dysfunction Score (P-MODS): development and validation of an objective scale to measure the severity of multiple organ dysfunction in critically ill children. Crit Care Med 2005; 33(7):1484-1491.

20. Leteurtre S, Martinot A, Duhamel A, Proulx F, Grandbastien B, Cotting J, et al. Validation of the paediatric logistic organ dysfunction (PELOD) score: prospective, observational, multicentre study. Lancet 2003;362(9379):192-197.

21. Rubenfeld GD. Improving clinical trials of long-term outcomes. Crit Care Med 2009;37(1 Suppl):S112-S116.

22. Pollack MM, Holubkov R, Glass P, Dean JM, Meert KL, Zimmerman J, et al. Functional Status Scale: new pediatric outcome measure. Pediatrics 2009;124(1):e18-e28.

23. Menikoff J. The paradoxical problem with multiple-IRB review. N Engl J Med 2010;363(17):1591-1593.

24. Curley MA, Arnold JH, Thompson JE, Fackler JC, Grant MJ, Fineman $\mathrm{LD}$, et al. Clinical trial design-effect of prone positioning on clinical outcomes in infants and children with acute respiratory distress syndrome. J Crit Care 2006;21(1):23-32; discussion 32-37.

25. Macrae D. Conducting clinical trials in pediatrics. Crit Care Med 2009;37(1 Suppl):S136-S139.

26. Jansen TC, Kompanje EJ, Bakker J. Deferred proxy consent in emergency critical care research: ethically valid and practically feasible. Crit Care Med 2009;37(1 Suppl):S65-S68. 
27. Randolph AG. The unique challenges of enrolling patients into multiple clinical trials. Crit Care Med 2009;37(1 Suppl):S107-S111.

28. National Institutes of Health. NIH policy and guidelines on the inclusion of children as participants in research involving human subjects. http://www.nichd.nih.gov/research/supported/cpccrn.cfm. Accessed June 20, 2011.

29. Schoenfeld DA, Hui Z, Finkelstein DM. Bayesian design using adult data to augment pediatric trials. Clin Trials 2009;6(4):297-304.

30. Melot C. New designs for clinical trials. Crit Care Med 2009;37(1 Suppl):S59-S64.

31. Halpern SD, Randolph AG, Angus DC. No child left behind: Enrolling children and adults simultaneously in critical care randomized trials. Crit Care Med 2009;37(9):2638-2641.

32. Flori HR, Ware LB, Milet M, Matthay MA. Early elevation of plasma von Willebrand factor antigen in pediatric acute lung injury is associated with an increased risk of death and prolonged mechanical ventilation. Pediatr Crit Care Med 2007;8(2):96-101.

33. Flori HR, Ware LB, Glidden D, Matthay MA. Early elevation of plasma soluble intercellular adhesion molecule-1 in pediatric acute lung injury identifies patients at increased risk of death and pro- longed mechanical ventilation. Pediatr Crit Care Med 2003;4(3): 315-321.

34. Angus DC, Mira JP, Vincent JL. Improving clinical trials in the critically ill. Crit Care Med 2010;38(2):527-532.

35. Meert KL, Donaldson AE, Newth CJ, Harrison R, Berger J, Zimmerman J, et al. Complicated grief and associated risk factors among parents following a child's death in the pediatric intensive care unit. Arch Pediatr Adolesc Med 2010;164(11):1045-1051.

36. Meert KL, Sarnaik AP. Choosing between death at home or in the hospital: respecting the principle of autonomy. Pediatr Crit Care Med 2010;11(3):438-439.

37. Meert KL, Briller SH, Schim SM, Thurston CS. Exploring parents' environmental needs at the time of a child's death in the pediatric intensive care unit. Pediatr Crit Care Med 2008;9(6):623-628.

38. Nathan DG. The several Cs of translational clinical research. J Clin Invest 2005;115(4):795-797.

39. Mendelsohn ME, Balke CW, Bonow RO, Fuster V, Grant AO, Mark DB, et al. Symposium presentations. J Am Coll Cardiol 2005;46(7 Suppl 1):A5-A70.

40. Dracup K, Bryan-Brown CW. From novice to expert to mentor: shaping the future. Am J Crit Care 2004;13(6):448-450.

\section{Discussion}

Fineman: In the physician-scientist academic model, do you think that the manner in which we support clinical scientists versus basic scientists is an issue, in terms of resources allocated and expectations for promotion? What I see, and hopefully it is changing, is that recruitment of a basic scientist comes with hundreds of thousands of dollars in lab space and equipment as a start-up package, yet you recruit a clinical scientist and you give them an office and a computer. In addition, in terms of promotability, you can do a tremendous amount of work and get your name on a very important clinical paper and you come to a promotion committee and it's, "Oh, she's the middle author on a paper," versus the basic scientist who in the same amount of time can be much more productive in terms of publications. Do you see this as a problem or an issue? Is it getting better?

Curley: I agree with you. I think we have to change some of the expectations, particularly in large clinical trials. Yes, they're formed around one specific aim, but in reality, if you build multiple questions within larger clinical trials and invite people to take the lead and own different pieces of data, they can be first author on what they've taken the lead on. A good example is the RESTORE trial; we have people who are focusing on weaning, people who are focusing on methadone, and they're going to own that piece of the study. We've also added several ancillary studies, so that's more data that can be used to give more people an opportunity to build their CV [curriculum vitae]. Definitely it's a problem with who's first and who's second within the larger paper, but I think you could build a whole bunch of secondary papers.

Willson: I think medicine has increasingly been hijacked by the business people. No offense to the business people, but we spend so much time on billing and how to maximize clinical revenue. I wish I had that time to spend on research. I think we need to reorient and remind people that our clinical care should be aimed at advancing research.

The oncologists follow that approach. When I was a resident, acute lymphocytic leukemia had $80 \%$ mortality, and now it's like catching a cold. They accomplished this by continuing attention to research; their whole attitude is one of research. I don't know why we spend so much time worrying about billing and clinical revenues when we could accomplish what oncologists have accomplished in critical care by educating patients' parents about the need for research, and, in many ways rescue clinical care from the business model.

Curley: It's interesting. When a child has cancer, they can't be treated unless they're on protocol. If we set it up in a similar way, we may learn a lot in a short period of time-for example, by saying to families, "We're a researchintensive facility and we want to learn from this horrible thing that has happened to your child."

I'd say it's grants management offices that have highjacked clinical research. To get a grant through the IRB is one thing, but to get it through grants management is unbelievable, and that's where all the indirects are spent: running the business of clinical trials. I've had sites say, "We can't participate in your clinical trial because there's no profit margin." You can't expect to run negative all the time, but clinical research is different than bench research. Bench researchers need a lab and a whole bunch of support in the lab to make it happen, and in the clinical world the unit is the lab. Consenting parents takes time, collecting and extracting the data takes time, doing in 
terventions takes time, running the blood samples takes time. A little bit of lab work, but surely not the $60-80 \%$ indirects these institutions are pulling in.

DiBlasi: In a 2008 paper $^{1}$ you suggested that when people are conducting clinical trials involving ventilator support devices, a multidisciplinary team should consist of nurses, RTs [respiratory therapists], and physicians. What key elements can RTs add to a clinical trial?

1. Irving SY, Curley MA. Challenges to conducting multicenter clinical research: ten points to consider. AACN Adv Crit Care 2008;19(2):164-169.

Curley: First of all, I've learned from RTs how to manage patients with ALI. I was trained by John Thompson and his group to pay attention to different things at the bedside. So if I went in as a nurse with my nursing lens and ignored the interface between the patient and the ventilator, my research would be fundamentally flawed because I didn't do it within the context of what was really happening to the patient.

For example, RTs I've worked with have built the weaning trials that can be generalizable in the real world; they contribute intellectual capital we need to make things happen. You can't just walk in and give surfactant: you have to look at the patient where they are and figure out a way to get it in and have it stay in. It's the nuances of running a protocol where RTs can enrich the team.

It has to be done in the multidisciplinary way. I think the problems we're having with tight glucose control have to do with the fact that no one's really figured out how to manage it. How is nutrition managed? You need to have a nutritionist involved. Team science is what we need, and we haven't done that consistently.

Brown: In a large trial at an institution I worked at, no RTs were consulted in the design of the study, and when they went to publish the study, they got rejected for design flaws that an RT would have pointed out. It was quite a shame. Eventually they got it published, but the study had to be portrayed negatively to get it published. I've seen that study referenced multiple times, and it's a shame because I think the intervention was actually positive.

Curley: Once you have the protocol developed, it should be the responsibility of the RTs to publish the respiratory therapy aspect of the protocol. That's where we don't do a good job. Different disciplines can take the lead with their questions and publish their piece of it in the journals that their colleagues read.

Wiswell: I want to put in my two cents about international investigators. I've been involved in a couple trials that enrolled large numbers of patients and had co-investigators in eastern Europe, western Europe, and South America. What I found is that a lot of international investigators are incredibly motivated, although they may not have done a lot of research. It can be less expensive to do research in their countries, and it can be of high quality. Investigators are well trained. You can also get research done in a timely fashion.

I think all of us are troubled by the research hurdles you brought up, and I think number one is the concern that as soon as you start a trial, the disease disappears. An international investigation increases the number of patients you can get. Granted, there will be more variables than if it's done in just one country or area, but I think with a lot of disorders you can do it successfully.

Curley: You could also capitalize on viral seasons: go to South America, collect data, then come to North America and follow the viral patterns. But make sure that the standards of care are comparable in participating units. We have lots of random variation in the United States, and I think there's probably better standards outside the United States, but you have to make sure everybody's practice is the same.

Willson: I was smiling as I heard Tom say that. Here's an example of an unanticipated problem we had in an international study. I was looking at surfactant in both adults and kids, and I had to travel to Korea because there were some questions about their results and they had a high mortality rate. Five of the subjects they enrolled with direct lung injury died of disseminated TB [tuberculosis]. We did not consider $\mathrm{TB}$ as a direct lung injury, but it is a pneumonia, so it met the definition. We just don't see it very often in the United States. So there can be unanticipated problems, but I couldn't agree more. We have collaborators in Israel, Australia, New Zealand, and Korea, and the level of care they give is certainly comparable to what we do here. It's true that you have to be careful. And it is complicated with time differences and things like getting drugs into the country, but it's all doable and it's actually a lot of fun.

Rubin: I have some comments for the readers of the Journal. This room is packed with critical care people, but there are a lot of opportunities for doing respiratory care research outside of the critical care unit. And once you move away, a lot of the problems you talk about aren't as important. Critical care involves episodic conditions that are incredibly severe, the family is under stress, it's difficult to get consent or assent when the subject is so ill and you don't have a long-term relationship prior to enrollment. The PICU is a different place; we are striving to fix things, get the patients better, and get them out. This can all make organizing research especially difficult.

Outside of the ICU there are great opportunities for research. For example, the Cystic Fibrosis Foundation has a therapeutic development network and most of the patients followed in cystic fibrosis centers are involved, 
similar to oncology studies. The cystic fibrosis physicians, therapists, and nurses are all engaged, and the Cystic Fibrosis Foundation has invested in training therapists and nurses to be investigators. The American Respiratory Care Foundation is revising their grant awards to try to develop more focused grants for training the next generation of respiratory therapy investigators. The future does look promising.

\section{Curley: Thank you.}

Cheifetz: Martha, I would like to discuss potential links to the adult ALI population. ${ }^{1}$ ALI/ARDS is a small component of what we see in pediatrics. Neonates aside for the moment, how do we link and partner with adult clinical trials to augment enrollment numbers and to potentially share experience, resources, and funding? The PALISI Network ${ }^{2-4}$ attempted to partner with the ARDS Network, but due to some logistical issues it was not very successful.

1. Randolph AG, Wypij D, Venkataraman ST, Hanson JH, Gedeit R, Meert KL, et al. Effect of mechanical ventilator weaning protocols on respiratory outcomes in infants and children: a randomized controlled trial. JAMA 2002;288(20):2561-2568.

2. Randolph AG, Meert KL, O’Neil ME, Hanson JH, Luckett PM, Arnold JH, et al; Pediatric Acute Lung Injury and Sepsis Investigator's (PALISI) Network. The feasibility of conducting clinical trials in infants and children with acute respiratory failure. Am J Respir Crit Care Med 2003; 167(10):1334-1340.

3. Randolph AG, Forbes PF, Gedeit RG, Arnold JH, Wetzel R, Luckett PM, et al; Pediatric Acute Lung Injury and Sepsis Investigator's (PALISI) Network. Cumulative fluid intake minus output is not associated with ventilator weaning duration or extubation outcomes in children. Pediatr Crit Care Med 2005;6(6):642-647.

4. Santschi M, Jouvet P, Leclerc F, Gauvin F, Newth CJ, Carroll CL, et al; Pediatric Acute Lung Injury and Sepsis Investigators Network (PALISI); European Society of Pediatric and Neonatal Intensive Care (ESPNIC). Acute lung injury in children: therapeutic practice and feasibility of international clinical trials. Pediatr Crit Care Med 2010;11(6):681-689.
Curley: PALISI has linked with ARDS Network, and I think we can continue to be successful there. They shared a lot of their expertise with us, and I think they continue to be receptive. In the past we automatically didn't cross the age chasm. Obviously there are differences between kids and adults, but where there isn't a physiologic difference in what is under study, the NIH has been very clear that we ought to enroll children. If you really want to study the phenomenon differently in children, you're going to have to enroll the infant population. Pediatric patients also are in NICUs [neonatal ICUs]. We have to stop thinking about geography and address these system issues. The people who screen patients should go to the NICU and to the adult ICU. The statistical stuff is interesting because it may be that you get most of your power from the adult population, but only if it makes sense. If it doesn't make developmental sense, we don't consider it. And if you can't even figure out what the grant application is saying, you should not do it; if it's statistically dense, you may not want to go there. If you can't eventually sell it to the people who are taking care of patients, because they don't understand what was done in the study, then you're not going to have generalizability and clinicians will not adopt your findings.

Cheifetz: Doug, how were you successful in integrating with our colleagues in the adult world for your surfactant trial?

Willson: I always take the attitude that adults are just big kids. No, I was just fortunate, Ira, that we started in pediatrics, and of course there are a lot more adults with respiratory failure than kids, and a lot more adults than kids. The company that sponsored the study was interested in getting into the adult world, because that's where they would see the greatest profit. They were generous in the sense that the most recent study continues to support the pediatric aspect, but their primary focus was the adult study. We were just lucky in that regard. The president of the company basically said, "We'll do kids because that's how we got here."

I think that kids down to the first year of life are more like adults than anything else. It's the neonates and infants that are probably different, and maybe that's where the dividing line should be. The FDA considers adults anybody 12 years or older. Adult funding should look at including kids, and actually many of them do, but it's often a halfhearted effort.

Gentile: Martha, the problem is the PICU. Now, let me explain the comment. The NICU is certainly a homogeneous population, with, for the most part, similar diseases. The adults groups are separated by age and body size. The PICU has neither of those two distinctive groups. Beside the range of patient ages (newborn to 18 years), the body weight and size requires understanding of both adult and neonatal practice. The PICU has made strides in many areas, such as HFOV [high-frequency oscillatory ventilation], ECMO [extra corporeal membrane oxygenation], and nitric oxide, that can be translated to care of adult patients. However, these are standard practice in the PICU, so it's difficult to include them in a randomized controlled trial where the patient may be selected to be part of a group receiving interventions such as HFOV, ECMO, or nitric oxide.

Curley: Sometimes clinicians who care for adult patients don't even consider solid pediatric papers when they design their studies. There are things we do well in pediatrics, and it's frustrating when I see that that whole body of literature is never considered For example, a bunch of pediatric people have been doing HFOV for a long time. Maybe it wasn't precisely researched, 
but they are some pediatric data that could be considered through the adult lens. We've contributed solidly in some areas, and it's unfortunate when those studies are not considered.

Brown: This is a point of contention with me. I've been a clinical specialist in the NICU and the PICU, and also worked in the adult world, and it's amazing to me the disconnect there: like these different places don't exist. An example of that is not looking at all the literature, as you mentioned, Martha. A recent example is in the NICU, where there is a trend towards not using saline for suctioning because of VAP. Then quite recently a very well designed adult study found that saline decreased VAP - the exact opposite of the current neonatal hypothesis. ${ }^{1}$ When I approached a leading neonatologist about investigating this, he told me, "No, those are adults." $\mathrm{He}$ won't even look at it. And I'm thinking, we're the same species, right? I was dumbfounded that we're going to continue down a path without even looking at a great study done in adults.

1. Caruso P, Denari S, Ruiz SA, Demarzo SE, Deheinzelin D. Saline instillation before tracheal suctioning decreases the incidence of ventilator-associated pneumonia. Crit Care Med 2009;37(1):330-322.
Curley: When we designed the VAP bundle, ${ }^{1}$ we had to look at the pediatric stuff and interpret all the literature in adults and neonates and come up with a recommendation for best evidence. I think you can extrapolate, but as soon as you lay it out, you're then obligated to build the evidence to support those recommendations.

1. Curley MA, Schwalenstocker E, Deshpande JK, Ganser CC, Bertoch D, Brandon J, et al. Tailoring the Institute for Health Care Improvement 100,000 Lives Campaign to pediatric settings: the example of ventilator-associated pneumonia. Pediatr Clin North Am 2006;53(6):1231-1251.

This article is approved for Continuing Respiratory Care Education credit. For information and to obtain your CRCE

(free to AARC members) visit

www.RCJournal.com 